$18,12,13$

\title{
Зависимость подвижности носителей заряда в гибридных наноструктурах на интерфейсе графена с молекулярными ионами от их зарядовой плотности
}

\author{
(C) А.В. Бутко, В.Ю. Бутко , Ю.А. Кумзеров \\ Физико-технический институт им. А.Ф. Иофффе РАН, \\ Санкт-Петербург, Россия \\ ฯ E-mail: vladimirybutko@gmail.com
}

Поступила в Редакцию 11 июня 2021 г.

В окончательной редакции 11 июня 2021 г.

Принята к публикации 12 июня 2021 г.

Гибридные наноструктуры с развитым интерфейсом между наноструктурными компонентами играют важную роль в современной электронике. В том числе, гибридные наноструктуры, формируемые на интерфейсе графена с ансамблями молекулярных ионов в графеновых полевых транзисторах (GFETs) c жидкими затворными изоляторами, перспективны для создания химических и биологических сенсоров. Поэтому изучение влияния интерфейса на электрический транспорт в таких системах представляет большой интерес. Настоящая работа направлена на теоретическое исследование зависимости подвижности носителей заряда $(\mu)$ в таких наноструктурах от плотности интерфейсных молекулярных ионов $\left(N_{\mathrm{ii}}\right)$. Установлено, что зависимость $\mu \propto 1 /\left(N_{\mathrm{ii}}\right)^{1 / 2}$, полученная в модели свободных носителей заряда в графене при условии их слабой связи с интерфейсными ионами для короткодействующих сил рассеяния, удовлетворительно описывает экспериментальные транзисторные характеристики при высоких напряжениях на затворе.

Ключевые слова: графен, гибридные наноструктуры, транзисторы, подвижность, интерфейс.

DOI: 10.21883/FTT.2021.11.51603.141

\section{1. Введение}

Интерес к исследованиям влияния интерфейса на электрический транспорт в гибридных наноструктурах, формируемых на границе графена с ансамблями молекулярных ионов в GFETs с затворными изоляторами в виде ионных растворов, в первую очередь, связан с важностью таких наноструктур для создания химических и биологических сенсоров [1-12]. Несмотря на этот интерес, в настоящее время, в описании электрического транспорта в указанных наноструктурах существует ряд нерешенных проблем. В том числе, к настоящему моменту исследовательские группы не пришли к однозначному выводу о зависимости подвижности носителей заряда $(\mu)$ в графене от плотности Кулоновских рассеивающих центров. Например, в работах [13] и [14] получены различные параметрические зависимости для подвижности носителей заряда от концентрации носителей заряда в графене $\left(\mu \propto 1 /(n)^{0.3}[13]\right.$ и $\left.\mu \propto 1 / n[14]\right)$, соответственно. Поэтому помимо указанных применений, является актуальным использование таких систем в качестве модельного объекта для установления закономерностей двумерного транспорта носителей заряда в наноструктурах при наличии Кулоновских рассеивающих центров. Для этой цели потенциальным достоинством рассматриваемых GFETs является возможность плавного изменения в широких пределах плотности интерфейсных ионов $\left(N_{\text {ii }}\right)$ (рассеивающих центров) с помощью управляющего напряжения на затворе $\left(V_{g}\right)$. Та- кая возможность отсутствует в образцах традиционных материалов, для каждого из которых плотность примесных ионов фиксирована. Поэтому в настоящей работе мы используем транзисторные характеристики GFETs c затворными изоляторами в виде водных растворов (см. рис. $1, a$ и $b$ ) для исследования зависимости подвижности носителей заряда от плотности рассеивающих центров. Мы также проводим сопоставление результатов, полученных на основе имеющихся в литературе экспериментальных данных, описывающих транспорт носителей заряда в графеновых полевых транзисторах с затворными изоляторами на основе водных растворов, с выводами простых теоретических моделей.

\section{2. Модель двумерного транспорта носителей заряда в GFETs при наличии интерфейсных ионных рассеивающих центров}

Двумерный электрический транспорт в приближении свободных носителей заряда в общем случае определяется различными видами их рассеяния. Для графена хорошо исследованными являются случаи рассеяния носителей заряда на фононах [15] и на примесных ионах [16]. Для высококачественных образцов графена при комнатной температуре вкладом этих видов рассеяния в ограничение проводимости часто можно пренебречь. В числе других механизмов рассеяния носителей 
заряда, вызывающих особый интерес в GFETs, можно назвать их рассеяние на других носителях заряда [17] и на дипольных флуктуациях интерфейсных графену квазипериодических ионных структур в жидких ионных затворных изоляторах [18]. Правильность выбора той или иной модели рассеивающих центров для конкретного случая определяется соотношением параметров выбранной задачи. В настоящей работе мы исследуем случай рассеяния носителей заряда в GFETs с затворными изоляторами в виде деионизированной воды, для которой концентрация молекулярных ионов значительно меньше, чем в ионных жидкостях, использованных в работе [18]. Поэтому эффекты формирования квазипериодических плотных ионных структур на поверхности графена [18] нами не рассматриваются. В то же время, учет влияния интерфейсных ионов на подвижность носителей заряда в графене необходим. Об этом косвенно свидетельствует влияние на проводимость графена эффектов памяти состояния, связанных с формированием на его поверхности квазистационарных ионных комплексов [9]. Поэтому в предлагаемой модели предполагается, что рассеяние свободных носителей заряда в графене
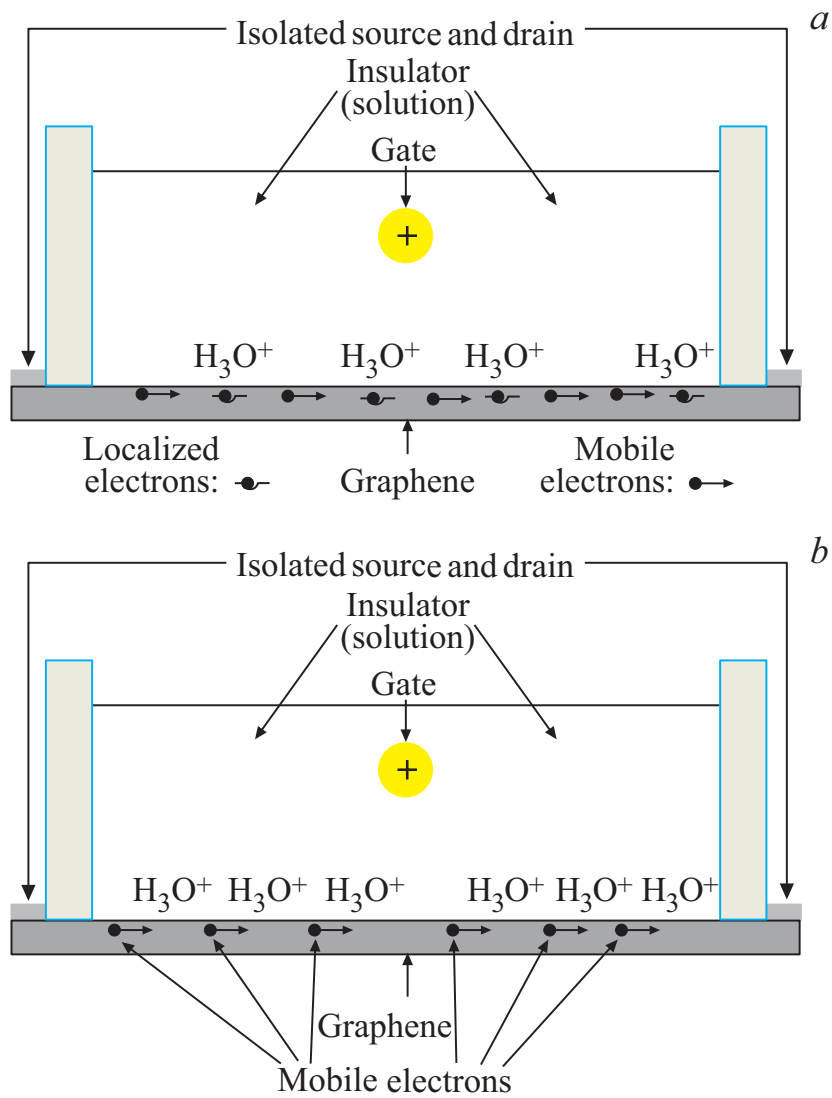

Рис. 1. $a-$ схематическое изображение GFET с водным затворным изолятором для случая сильной связи между положительными интерфейсными ионами и электронами. $b-$ схематическое изображение GFET с водным затворным изолятором для случая слабой связи между положительными интерфейсными ионами и электронами.
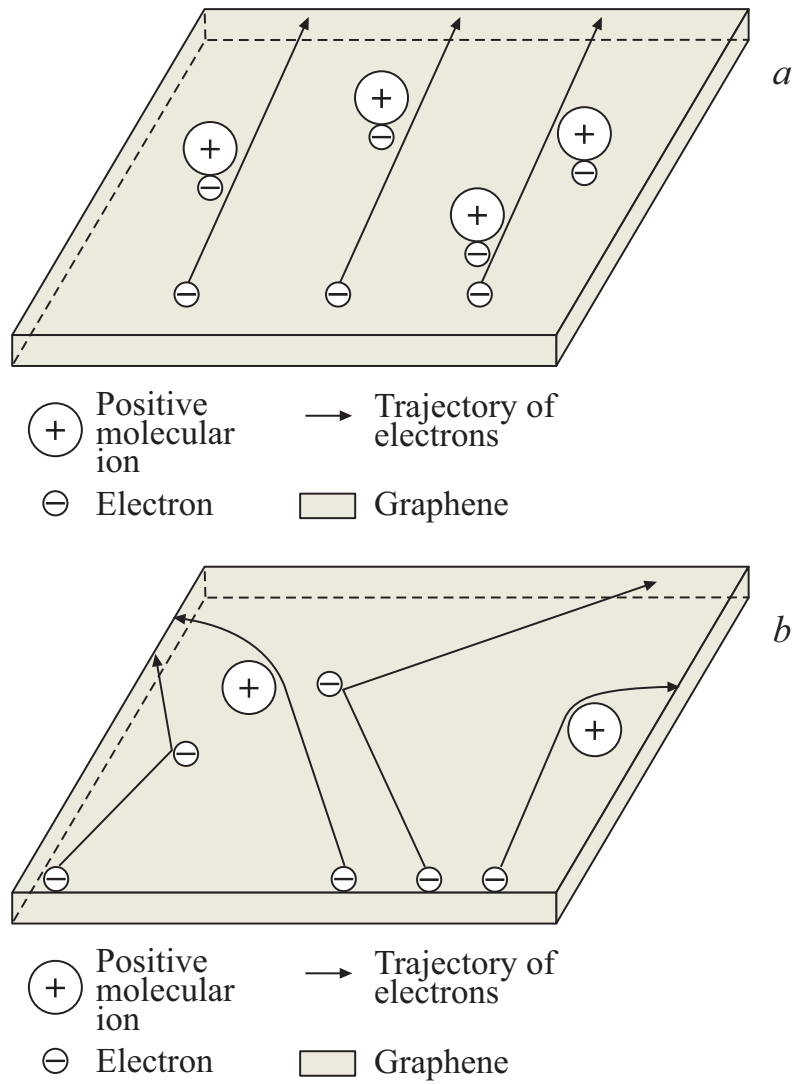

Рис. 2. $a-$ схематическое изображение транспорта электронов в GFET с жидким затворным изолятором для случая сильной связи между положительными интерфейсными ионами и электронами. $b-$ схематическое изображение транспорта электронов в GFET с жидким затворным изолятором для случая слабой связи между положительными интерфейсными ионами и электронами.

может происходить не только на свободных носителях заряда (их двумерная (2D) плотность: $n$ ), но и на интерфейсных молекулярных ионах. При таком рассмотрении возникают два предельных случая, которые мы будем называть случаем сильной связи и случаем слабой связи.

В первом из них, (случай сильной связи) благодаря сильному кулоновскому притяжению свободные носители заряда противоположного знака настолько близко расположены к любому из интерфейсных ионов (см. рис. $1, a$ и рис. $2, a)$, что происходит практически полное экранирование электрического поля интерфейсных ионов, и вкладом в рассеяние свободных носителей заряда от создаваемых при этом диполей можно пренебречь. Благодаря полевому эффекту $n \approx n_{0}+N_{\mathrm{ii}}$, где $n_{0}$ - равновесная плотность носителей заряда в графене в отсутствии напряжения на затворе. Поэтому для рассматриваемого случая вклады в рассеяние свободных носителей заряда от электрических полей интерфейсных ионов и от электрических полей свободных носителей заряда, инжектированных в графен благодаря полевому 
эффекту, взаимно компенсируют друг друга, приводя к практически полной независимости подвижности носителей заряда от плотности интерфейсных ионов и затворного напряжения.

Во втором предельном случае слабой связи (см. рис. $1, b$ и рис. $2, b)$, Ферми энергия или тепловая энергия свободных носителей заряда достаточно велика, чтобы препятствовать их локализации (стационарному сближению с интерфейсными ионами). При этом не происходит полной компенсации электрических полей, создаваемых интерфейсными ионами и свободными носителями заряда, и вклады в рассеяние от этих полей необходимо учитывать независимо при определении подвижности носителей заряда. Оба эти вклада уменьшают среднее время свободного пробега носителей заряда $(\tau)$.

Таким образом, путем изучения экспериментальных зависимостей подвижности носителей заряда от плотности интерфейсных ионов и затворного напряжения возможно определение того, какой из этих предельных случаев лучше соответствует эксперименту.

Проводимость графена $(\sigma)$ может быть оценена с помощью следующей формулы: $\sigma \approx e^{2} \cdot n \cdot \tau /(2 m)$, где $e$ - заряд электрона, $n$ - плотность свободных носителей заряда в графене, $m$ - масса свободного носителя заряда, которую мы в дальнейшем будем считать равной массе электрона. В предположении, что причиной рассеяния свободных носителей заряда являются частично экранированные Кулоновские короткодействующие силы (короткодействующие по сравнению с длиной свободного пробега), длина свободного пробега носителей заряда в графене $(\lambda)$ дается следующей формулой: $\lambda \approx \tau v_{\mathrm{F}}$, где $v_{\mathrm{F}}-$ Ферми скорость носителей заряда в графене. Время свободного пробега $\tau$ для случая слабой связи дается формулой: $1 / \tau=1 / \tau_{\mathrm{fc}}+1 / \tau_{\mathrm{ii}}$, где $\tau_{\mathrm{ii}}$ - среднее время свободного пробега носителей заряда до рассеяния на интерфейсных ионах и $\tau_{\mathrm{fc}}-$ среднее время свободного пробега носителей заряда до их рассеяния на других свободных носителях заряда. Подвижность носителей заряда при этом дается формулой $\mu \approx e \cdot \lambda /\left(2 m \cdot v_{\mathrm{F}}\right)$. Полагая $v_{\mathrm{F}}$ постоянной величиной, основная зависимость подвижности носителей заряда от $N_{\text {ii }}$ определяется зависимостью от нее длины свободного пробега. Для двумерного случая длина свободного пробега носителей заряда между рассеяниями на интерфейсных ионах $\lambda_{\mathrm{ii}}=\tau_{\mathrm{ii}} v_{\mathrm{F}} \propto 1 /\left(N_{\mathrm{ii}}\right)^{1 / 2}$. При высоких затворных напряжениях, благодаря полевому эффекту, выполняется соотношения $n \approx N_{\mathrm{ii}}$. Для этого случая длина свободного пробега носителей заряда между рассеяниями на других свободных носителях заряда дается выражением $\lambda_{\mathrm{fc}}=\tau_{\mathrm{fc}} v_{\mathrm{F}} \propto 1 /\left(N_{\mathrm{ii}}\right)^{1 / 2}$. Таким образом, в предположении слабой связи, используя выражение $1 / \lambda=1 / \lambda_{\mathrm{fc}}+1 / \lambda_{\mathrm{ii}}$, получаем $\lambda \propto 1 /\left(N_{\mathrm{ii}}\right)^{1 / 2}$ и, соответственно, зависимость подвижности носителей заряда от интерфейсной зарядовой плотности определяется формулой: $\mu \propto 1 /\left(N_{\mathrm{ii}}\right)^{1 / 2}$.

\section{3. Сопоставление экспериментальных и теоретических результатов}

Полученные зависимости подвижности носителей заряда в графене от плотности интерфейсных ионов в GFETs нуждаются в экспериментальной проверке. C этой целью мы использовали экспериментальные данные о проводимости графена в GFETs с затворным изолятором в виде деионизированной воды, опубликованные в работе [7]. Схематические изображения использованного в этой работе GFET представлены на рис. $1, a$ и рис. $1, b$. На рис. 3, $a$ представлена зависимость проводимости для двух различных измерений
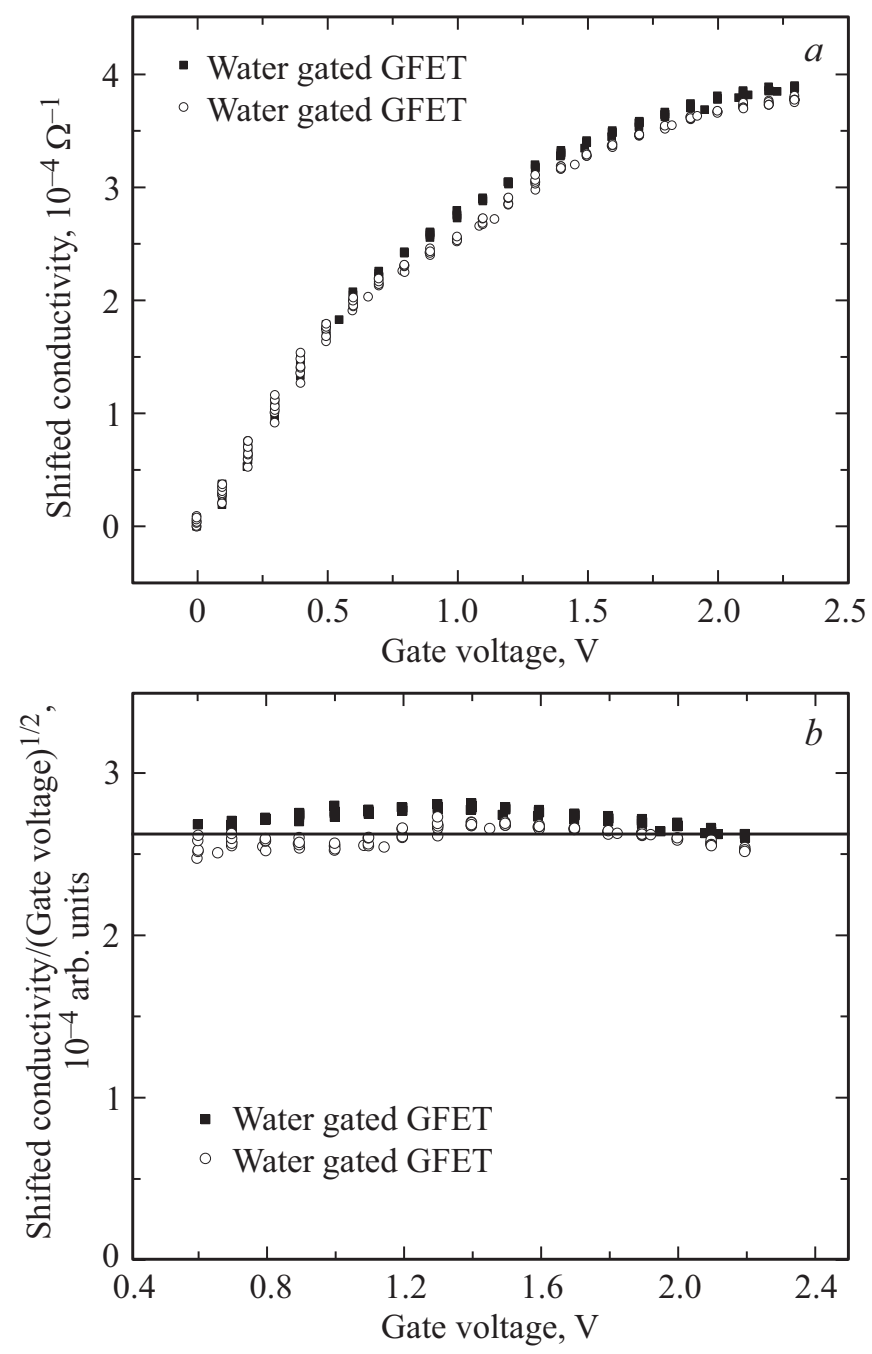

Рис. 3. $a-$ результаты измерений зависимости проводимости GFET от напряжения на затворе, выполненных в работе [7], после смещения затворного напряжения на $0.5 \mathrm{~V}$ и вычитания из измеренной проводимости ее значения в минимуме кривой (в точке Дирака). $b$ - данные, полученные в результате деления значений проводимости, представленных на рис. 3, $a$, на $V_{g}^{1 / 2}$. Горизонтальная линия является вспомогательным построением для наглядного подтверждения слабой зависимости полученной величины в представленном диапазоне напряжений. 
от напряжения на затворе. При построении данной зависимости нами был осуществлен сдвиг $+0.5 \mathrm{~V}$ по напряжению. Кроме этого, было осуществлено вычитание значения проводимости графена в точке Дирака, для совмещения этой точки с началом отсчета. Мы предполагаем, что при высоких напряжениях на затворе независящая от плотности интерфейсных ионов емкость двойного слоя $\left(C_{\mathrm{dl}}\right)$ много меньше квантовой емкости $\left(C_{\mathrm{q}}\right)$, которая, как известно [19], возрастает при увеличении плотности носителей заряда $\left(C_{\mathrm{dl}} \propto(n)^{1 / 2}\right)$. При этом условии напряжение, прикладываемое к затвору, прямо пропорционально интерфейсной плотности молекулярных ионов на поверхности графена $\left(V_{g} \propto N_{\text {ii }}\right)$. (Коэффициент пропорциональности при этом зависит от $\left.C_{\mathrm{dl}}\right)$. С учетом справедливого при высоких уровнях напряжения на затворе соотношения $n \approx N_{\mathrm{ii}}$, получаем, что $V_{g} \propto n$. Для сравнения предсказания нашей модели $\left(\mu \propto 1 / n^{1 / 2}\right)$ подставляем это выражение в формулу для проводимости $\sigma \approx e n \mu$, получаем, $\sigma \propto n^{1.2}$, а, следовательно, проводимость $\sigma \propto V_{g}^{1 / 2}$. На рис. $3, b$ представлен результат деления проводимости графена на корень квадратный из значения напряжения на затворе. Как видно из этого рисунка в результате такого деления получаем примерно постоянный уровень. Таким образом, предлагаемая модель удовлетворительно описывает зависимость подвижности носителей заряда от интерфейсной плотности молекулярных ионов для случая слабой связи. Для другого образца графена с худшей морфологией, исследовавшегося в той же работе [7], аналогичный подход дает зависимость $\mu \propto 1 / n^{0.3}$, что соответствует результатам работы [13]. Вероятно, большее число дефектов в этом образце, вносит независящий от $N_{\text {ii }}$ вклад в рассеяние свободных носителей заряда, тем самым ослабляя наблюдаемую зависимость от $N_{\mathrm{ii}}$.

\section{4. Заключение}

Для образцов высококачественного графена для случая слабой связи предлагаемая модель удовлетворительно описывает зависимость подвижности свободных носителей заряда от плотности интерфейсных ионов $\left(\mu \propto 1 /\left(N_{\mathrm{ii}}\right)^{1 / 2}\right)$. Для образцов графена с большим количеством дефектов наблюдается более слабая зависимость, чем предсказывает предлагаемая модель. Помимо большего вклада дефектов в рассеяние носителей заряда это обстоятельство может быть также обусловлено не вполне точным выполнением оценочных предположений, сделанных при получении такой зависимости.

\section{Благодарности}

Авторы благодарны В.Ю. Давыдову, А.А. Лебедеву, С.П. Лебедеву, И.А. Елисееву, А.А. Сысоевой и А.В. Фокину за помощь.

\section{Финансирование работы}

Исследование выполнено за счет гранта Российского научного фонда (проект № 21-72-20038).

\section{Конфликт интересов}

Авторы заявляют об отсутствии конфликта интересов.

\section{Список литературы}

[1] P.K. Ang, W. Chen, A.T.S. Wee, K.P. Loh. J. Am. Chem. Soc. 130, 44, 14392 (2008).

[2] H. Li, Y. Zhu, M.S. Islam, M.A. Rahman, K.B. Walsh, G. Koley. Sens. Actuators B Chem. 253, 759 (2017).

[3] M.H. Lee, B.J. Kim, K.H. Lee, I.-S. Shin, W. Huh, J.H. Cho, M.S. Kang. Nanoscale 7, 17, 7540 (2015).

[4] N.S. Green, M.L. Norton. Anal. Chim. Acta 853, 127 (2015).

[5] S. Taniselass, M.K.M. Arshad, S.C.B. Gopinath. Biosens. Bioelectron. 130, 276 (2019).

[6] X. You, J.J. Pak, Sens. Actuators B 202, 1357 (2014).

[7] A.V. Butko, V.Y. Butko, S.P. Lebedev, A.A. Lebedev, V.Y. Davydov, I.A. Eliseyev, Y.A. Kumzerov. J. Appl. Phys. 128, 21, 215302 (2020).

[8] A.V. Butko, V.Yu. Butko, S.P. Lebedev, A.A. Lebedev, Yu.A. Kumzerov. Phys. Solid State 60, 12, 2668 (2018).

[9] A.V. Butko, V.Y. Butko, S.P. Lebedev, A.A. Lebedev, V.Y. Davydov, A.N. Smirnov, I.A. Eliseyev, M.S. Dunaevskiy, Y.A. Kumzerov. Appl. Surf. Sci. 444, 36 (2018).

[10] A.V. Butko, V.Yu. Butko, S.P. Lebedev, A.A. Lebedev, Y.A. Kumzerov. Phys. Solid State 59, 10, 2089 (2017).

[11] A.V. Butko, V.Yu. Butko, S.P. Lebedev, A.N. Smirnov, V.Yu. Davydov, A.A. Lebedev, Yu.A. Kumzerov. Phys. Solid State 58, 7, 1483 (2016).

[12] A.V. Butko, V.Yu. Butko. Phys. Solid State 57, 5, 1048 (2015).

[13] M. Dankerl, M.V. Hauf, A. Lippert, L.H. Hess, S. Birner, I.D. Sharp, A. Mahmood, P. Mallet, J. Veuillen, M. Stutzmann, J.A. Garrido. Adv. Funct. Mater. 20, 18, 3117 (2010).

[14] J.L. Tedesco, B.L. VanMil, R.L. Myers-Ward, J.M. Mc Crate, S.A. Kitt, P.M. Campbell, G.G. Jernigan, J.C. Culbertson, C.R. Eddy, D.K. Gaskill. Appl. Phys. Lett. 95, 12, 122102 (2009).

[15] A. Browning, N. Kumada, Y. Sekine, H. Irie, K. Muraki, H. Yamamoto. Appl. Phys. Exp. 9, 6, 065102 (2016).

[16] S. Adam, E.H. Hwang, V.M. Galitski, S. Das Sarma. PNAS 104, 47, 18392 (2007).

[17] E.H. Hwang, S. Adam, S. Das Sarma. Phys. Rev. Lett. 98, 18, 186806 (2007).

[18] T.A. Petach, K.V. Reich, X. Zhang, K. Watanabe, T. Taniguchi, B.I. Shklovskii, D. Goldhaber-Gordon. ACS Nano 11, 8, 8395 (2017).

[19] J. Xia, F. Chen, J. Li, N. Tao. Nature Nanotechnology 4, 8, 505 (2009)

Редактор К.В. Емцев 